\title{
Influência do espaçamento em primeira e segunda rotação de eucalipto manejado sob regime de curta rotação
}

\author{
Vitor Moreira Guimarães ${ }^{1}$, Thelma Shirlen Soares ${ }^{2}$, Edmilson Santos Cruz ${ }^{3}$
}

DOI: https://doi.org/10.35699/2447-6218.2020.19247

\begin{abstract}
Resumo
Este estudo teve como objetivo avaliar características dendrométricas e propriedades da madeira do clone 1277 ( $E$. grandis $\mathrm{x}$ E. camaldulensis), em duas rotações, manejado em curta rotação em Jataí-GO. Utilizou-se o delineamento em blocos casualizados com seis espaçamentos (3,0 m x 0,5 m; 3,0 m x 1,0 m; 3,0 m x 1,5 m; 3,0 m x 2,0 m; 3,0 m x 2,5 m e 3,0 m x 3,0 m) e três repetições. Em cada rotação, avaliou-se: diâmetro a 1,30 m de altura do solo (DAP), altura total (HT), volume total (VT), densidade básica (DB), poder calorífico superior (PCS) e teores de carbono (C), hidrogênio $(\mathrm{H})$ e oxigênio $(\mathrm{O})$ presentes na madeira. Os dados foram analisados por meio da análise de variância e para efeitos significativos realizou-se o teste de Scott-Knott $(\alpha=5 \%)$. O teste t pareado $(\alpha=5 \%)$ foi empregado para comparação entre a primeira e a segunda rotação, avaliando-se as características em cada espaçamento. Na primeira rotação, apenas o DAP e VT foram influenciados pelos espaçamentos enquanto que, na segunda rotação, os espaçamentos influenciaram o DAP, o VT e os teores de C, O e H. Em ambas rotações, o DAP foi maior nos espaçamentos mais amplos com maior produção volumétrica ocorreu no espaçamento mais adensado. Comparando as duas rotações, verificou-se, que para grande parte das características avaliadas, houve diferença significativa em todos os espaçamentos, com decréscimos em todas as variáveis dendrométricas e teores de $\mathrm{C}$ e $\mathrm{H}$ e acréscimo na DB, PCS e teor de $\mathrm{O}$.
\end{abstract}

Palavras-chave: Alto fuste. Talhadia. Características dendrométricas. Propriedades da madeira.

\section{Influence of spacing in first and second rotation of eucalyptus managed under system of short rotation}

\begin{abstract}
This study aimed to evaluate the dendrometric characteristics and wood properties of a two-rotation of the 1277 eucalypt clone (E. grandis $\mathrm{x}$ E. camaldulensis) in Jataí-GO, Brazil, with short rotation system. The experiment design used was randomized blocks with six planting density $(3.0 \times 0.5 \mathrm{~m}, 3.0 \times 1.0 \mathrm{~m} ; 3.0 \times 1.5 \mathrm{~m} ; 3.0 \times 2.0 \mathrm{~m} ; 3.0 \times 2.5$ $\mathrm{m} 3.0 \times 3.0 \mathrm{~m}$ ) and three replications. At each rotation, diameter at $1.30 \mathrm{~m}$ above ground level (DBH), total height (HT), total volume (VT), basic density (DB), higher heating value (HHC) and carbon (C), hydrogen (H) and oxygen (O) contents in wood were evaluated. The data obtained were analyzed using analisys of variance and for significant effects the Scott-Knott $(\alpha=5 \%)$ test was performed. The paired t-test $(\alpha=5 \%)$ was used for comparison between the first and second rotation, evaluating the characteristics at each spacing. In the first rotation, the spacing affect significantly the DBH and VT, while in the second rotation, the DBH, VT, and C, O and H contents were influenced by the spacing. In both rotations, the DBH showed the largest values in greater spacing while the VT showed the largest values in smaller spacings. Comparing the two rotations, it was verified that for most of the evaluated characteristics,
\end{abstract}

\footnotetext{
${ }^{1}$ Universidade Federal de Jatai, Curso de Engenharia Florestal, Câmpus Jatobá , Jataí, GO. Brasil. https://orcid.org/0000-0001-8408-2792

${ }^{2}$ Universidade Federal de Jatai, Curso de Engenharia Florestal, Câmpus Jatobá, Jataí, GO. Brasil. https://orcid.org/0000-0003-1532-5038

${ }^{3}$ Universidade Federal de Jatai, Curso de Engenharia Florestal, Câmpus Jatobá, Jataí, GO. Brasil. https://orcid.org/0000-0002-7503-3697

*Autor para correspondência: thelsoares@gmail.com
}

Recebido para publicação em 10 de fevereiro de 2020. Aceito para publicação em 25 de julho de 2020. e-ISSN: 2447-6218 / ISSN: 2447-6218. Atribuição CC BY. 
there was a significant difference in all spacing, with decreases in all dendrometric variables and $\mathrm{C}$ and $\mathrm{H}$ contents and increase in DB, PCS and O content.

Key words: High forest regime. Coppice regime. Dendrometric characteristics. Wood properties.

\section{Introdução}

Com uma área de 7,83 milhões de hectares de reflorestamento, o setor brasileiro de árvores plantadas é responsável por $91 \%$ de toda a madeira produzida para fins industriais e 6,9\% do PIB Industrial no País (Ibá, 2017). Ademais, é considerado como um dos segmentos com maior potencial de contribuição para a construção de uma economia verde (Ibá, 2019).

O cultivo de espécies florestais de rápido crescimento tem aumentado no Brasil devido à diversificação do uso de sua madeira e pela facilidade de adaptação às diferentes condições edafoclimáticas (Prevedello et al., 2013). Com isso, as florestas de eucalipto têm tomado espaços cada vez maiores no território nacional e tem sido usada como fonte energética, gerando energia limpa e renovável, evitando que seja extraída madeira de florestas nativas (Vechi e Magalhães Júnior, 2018). Além disso, essas espécies contribuem no sequestro de carbono, auxiliando na melhoria da qualidade do ar (Paixão et al., 2006).

O eucalipto é uma espécie que pode ser cultivada em quase todos os tipos de clima, possui crescimento rápido, alta capacidade de regeneração permitindo a condução de até três rotações (Scolforo, 2008) e colheita a partir de 2 anos quando o objetivo é produção de biomassa para geração de energia. Devido a sua grande plasticidade ambiental, altos índices de produtividade, ampla diversidade de espécies e características energéticas (densidade da madeira e poder calorífico), é o gênero mais utilizado para a implantação de florestamento e reflorestamentos com fins energéticos (Couto e Müller, 2008).

O termo florestas energéticas define os plantios florestais com a finalidade de produzir maior volume de biomassa por área em menor espaço de tempo contribuindo, seja pela produção de carvão vegetal ou pela queima direta da lenha, para o aumento de alternativas renováveis e sustentáveis que contribuem para diversificação da matriz energética brasileira. Estas florestas implantadas em sistemas adensados aproveitam melhor o uso do solo e produzem, relativamente, mais matéria seca do que os plantios convencionais (Macedo, 2003; Müller, 2005a). No manejo de florestas energéticas, têm-se adotado o uso de espaçamentos adensados, curta rotação ( 2 a 3 anos) e condução de rebrota.

A condução da brotação de cepas, após o corte raso da floresta, é aplicável a espécies florestais que têm capacidade de regenerar vegetativamente. Esse sistema de condução de povoamentos florestais, denominado talhadia, é adequado à maioria das espécies do gênero Eucalyptus, que apresentam a capacidade de lançar brotos em razão das gemas adventícias presentes no tronco. Tal característica é desejável, pois a partir de um único plantio pode-se obter uma ou mais colheitas, com redução significativa de gastos com reforma (Barros et al., 2002). Segundo Guedes et al. (2011), em algumas situações, a talhadia se torna uma opção que gera maior lucro, mas em alguns casos a regeneração de substituição pode ser a solução, em decorrência ao aumento do potencial de produção por meio do melhoramento genético.

A talhadia é o sistema silvicultural predominante nos países em desenvolvimento da África, América e Ásia para produção de lenha para uso social ou industrial, com tendência de ser de empregado nos países desenvolvidos para produção de biomassa para processos industriais (Evans e Turnbull, 2004). A talhadia apresenta a vantagem de reduzir os custos de produção, podendo ser uma alternativa viável para a silvicultura comercial (Crous e Burger, 2014), principalmente pra a produção de madeira para energia.

Considerando que no Brasil estudos que avaliam talhadia em curta rotação ainda são escassos e que a correta definição do espaçamento de plantio é um dos principais fatores envolvidos no manejo das florestas energéticas por influenciar o crescimento das árvores e a propriedade da madeira, este estudo teve como objetivo avaliar a influência de diferentes espaçamentos nas características dendrométricas e nas propriedades da madeira de um povoamento de eucalipto manejado em curta rotação, em primeira e em segunda rotação.

\section{Material e Métodos}

Os dados analisados pertencem a um experimento implantado na Regional Jataí (REJ) da Universidade Federal de Goiás (UFG), em Jataí-GO, localizada nas coordenadas $17^{\circ} 56^{\prime} \mathrm{S}$ e $51^{\circ} 43^{\prime} \mathrm{O}$. O clima da região é do tipo Aw classificado como mesotérmico, com estação seca e chuvosa (tipo Aw (Alvarez et al., 2013). O solo é classificado como Latossolo Vermelho-Escuro distroférrico (Embrapa, 2018).

O delineamento utilizado para o plantio foi o de blocos casualizados, com três repetições, considerando seis densidades de plantio ( $3,0 \mathrm{~m} \mathrm{x} 0,5 \mathrm{~m}, 3,0 \mathrm{~m} \mathrm{x} \mathrm{1,0} \mathrm{m}$; $3,0 \mathrm{~m} \times 1,5 \mathrm{~m} ; 3,0 \mathrm{~m} \times 2,0 \mathrm{~m} ; 3,0 \mathrm{~m} \times 2,5 \mathrm{~m} \mathrm{e} \mathrm{3,0} \mathrm{m} \mathrm{x} \mathrm{3,0}$ $\mathrm{m})$ distribuídos em parcelas de área variável $\left(27 \mathrm{~m}^{2}, 54\right.$ 
$\mathrm{m}^{2}, 81 \mathrm{~m}^{2}, 108 \mathrm{~m}^{2}, 135 \mathrm{~m}^{2}$ e $162 \mathrm{~m}^{2}$, respectivamente). Foram utilizadas mudas clonais do híbrido $E$. grandis $\mathrm{x} E$. camaldulensis (clone 1277), o qual é bastante utilizado na região Sudoeste do Estado de Goiás e caracteriza-se por ser tolerante a ambientes com deficiências hídricas. $\mathrm{Na}$ Figura 1 é apresentada a localização da área de estudo e o croqui experimental com o esquema da distribuição dos tratamentos em cada bloco.

Figura 1 - Localização da área de estudo e arranjo das parcelas com seus respectivos tratamentos, sendo: $1=3,0 \mathrm{x}$ $0,5 \mathrm{~m} ; 2=3,0 \times 1,0 \mathrm{~m} ; 3=3,0 \times 1,5 \mathrm{~m} ; 4=3,0 \times 2,0 \mathrm{~m} ; 5=3,0 \times 2,5 \mathrm{~m}$ e $6=3,0 \times 3,0 \mathrm{~m}$.

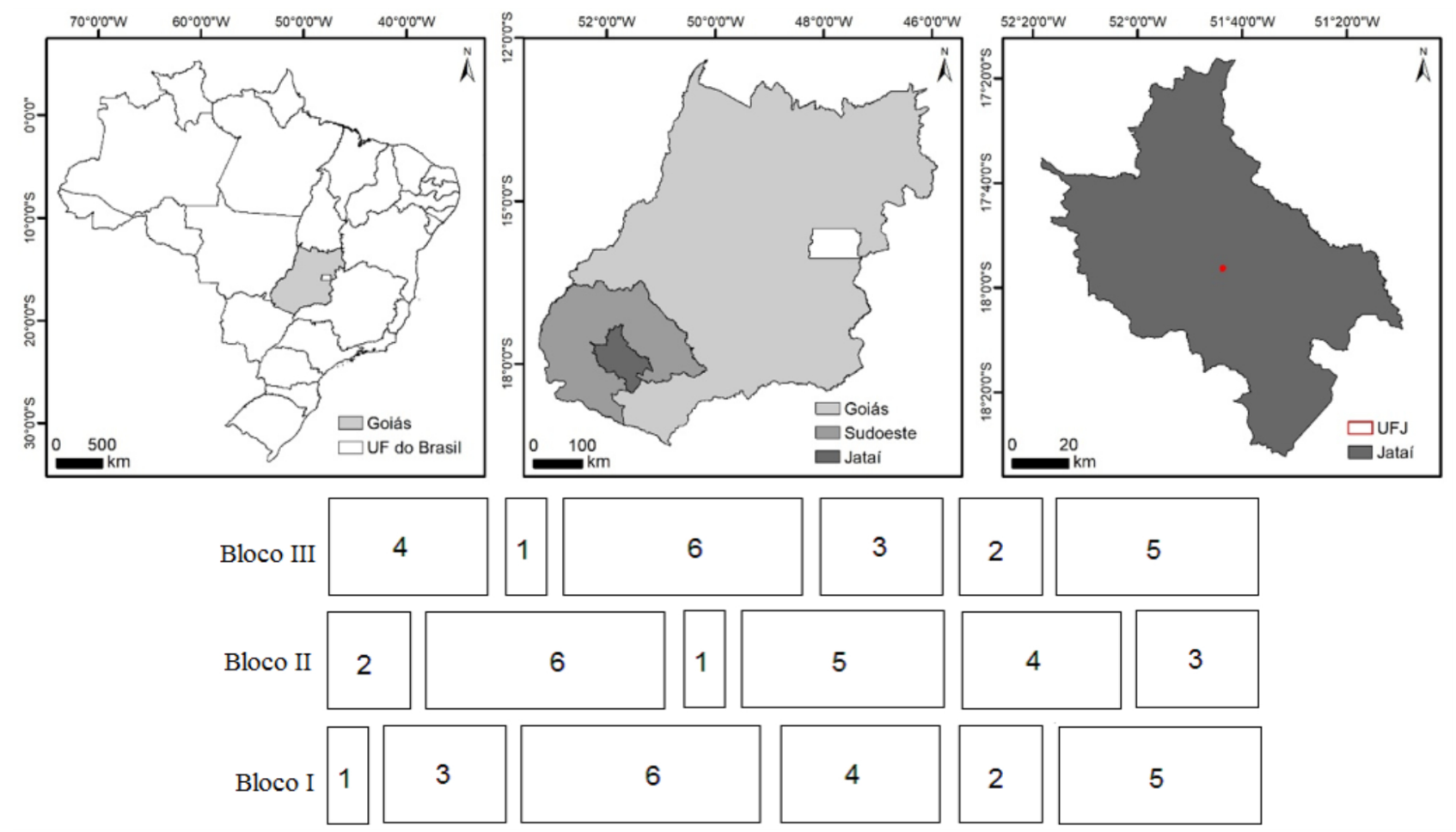

$\mathrm{Na}$ primeira rotação, o corte das árvores foi realizado deixando as cepas com $0,15 \mathrm{~m}$ de altura. Em seguida, iniciou-se a remoção dos galhos da área e início dos tratos silviculturais para a condução dos brotos. Seis meses após o início da condução dos brotos, foi realizada a desbrota em todas as árvores deixando um broto por cepa para condução da talhadia. As avaliações na primeira e na segunda rotação foram efetuadas quando o povoamento tinha 24 meses de idade.

Para cada árvore, foram mensurados o diâmetro a 1,30 m de altura do solo (DAP) e a altura total (HT). Assim também, em cada rotação, foi realizado o abate de uma árvore por parcela totalizando 18 árvores. A seleção das árvores que foram abatidas foi baseada na metodologia da árvore-modelo, a qual é definida em função do diâmetro médio quadrático segundo metodologia descrita por Campos e Leite (2017).

O volume total foi obtido por meio da cubagem das árvores-amostra as quais foram cubadas pelo método de Smalian medindo-se nas diferentes alturas 2,0; 4,0; $6,0 \mathrm{~m}$, e assim sucessivamente, até $5 \mathrm{~cm}$ de diâmetro do fuste com casca.

Para a obtenção da densidade básica e do poder calorífico superior, em cada árvore-amostra, foram retirados discos de 2,5 cm de espessura a 0\%, 25\%, 50\%,
$75 \%$ e $100 \%$ da altura comercial, considerada até um diâmetro mínimo de $5 \mathrm{~cm}$ com casca. A partir destes discos, foram retiradas amostras para a determinação da densidade básica pelo método de imersão segundo a NBR 11941 (ABNT, 2003). A densidade básica média da árvore foi considerada como sendo a média aritmética dos pontos de amostragem longitudinal no tronco das árvores, sem considerar a posição do DAP. A determinação da densidade básica foi realizada no Laboratório de Madeira e Materiais da REJ-UFG.

As quantificações do poder calorífico superior e teores de componentes elementares da madeira foram realizadas no Laboratório de Energia da Biomassa Florestal da Universidade Federal de Lavras. Para a determinação do poder calorífico superior, foram retiradas amostras nos discos visando a formação de uma amostra composta por árvore. O poder calorífico superior foi determinado em um calorímetro digital IKA C-200, conforme a norma NBR 8633 (ABNT, 1984).

Para a determinação dos componentes elementares (Carbono - C, Hidrogênio - H e Nitrogênio - N) da madeira foram retiradas amostras nos discos visando à formação de uma amostra composta por árvore-amostra. As amostras compostas foram trituradas e peneiradas, sendo utilizada a fração que passou pela peneira de 60 mesh e ficou retida pela peneira de 270 mesh conforme 
Guimarães, V. M. et al.

realizado no trabalho de Protásio et al. (2014). A quantificação dos teores de carbono e hidrogênio em relação à massa seca da madeira foi realizada em um analisador universal da marca Elementar (modelo Vario Micro Cube). O teor de oxigênio foi obtido por diferença em relação aos demais componentes elementares conforme Bech et al. (2019).

Informações sumarizadas sobre estatísticas descritivas das características avaliadas em cada rotação são apresentadas na Tabela 1.

Tabela 1 - Valores mínimo, médio, máximo e desvio padrão das características diâmetro a 1,30 m de altura do solo (DAP), altura total (HT), volume total (VT), densidade básica (DB), poder calorífico superior (PCS), teor de carbono $(\mathrm{C})$, teor de hidrogênio $(\mathrm{H})$ e teor de oxigênio $(\mathrm{O})$ para o clone 1277 em função do espaçamento em primeira (R1) e segunda rotação (R2).

\begin{tabular}{lccccccccccccccccc}
\hline & \multicolumn{2}{c}{ DAP } & \multicolumn{2}{c}{ HT } & \multicolumn{2}{c}{ VT } & \multicolumn{2}{c}{ DB } & \multicolumn{2}{c}{ PCS } & \multicolumn{2}{c}{ C } & H & \multicolumn{3}{c}{ O } \\
\cline { 2 - 15 } & R1 & R2 & R1 & R2 & R1 & R2 & R1 & R2 & R1 & R2 & R1 & R2 & R1 & R2 & R1 & R2 \\
\hline Mínimo & 8,1 & 4,5 & 11,9 & 6,9 & 80,1 & 39,6 & 0,4 & 0,5 & 4416,0 & 4533,0 & 48,1 & 40,8 & 6,5 & 4,9 & 40,7 & 43,9 \\
Médio & 10,8 & 7,4 & 13,2 & 11,4 & 157,8 & 102,8 & 0,5 & 0,5 & 4598,2 & 4678,5 & 48,6 & 45,0 & 6,6 & 5,6 & 42,1 & 48,6 \\
Máximo & 13,1 & 10,8 & 16,4 & 15,7 & 323,4 & 184,8 & 0,5 & 0,5 & 4804,5 & 4827,0 & 49,4 & 49,1 & 6,8 & 6,3 & 43,0 & 53,5 \\
Desvio & 1,3 & 2,0 & 1,0 & 2,5 & 78,5 & 45,4 & 0,0 & 0,0 & 101,6 & 78,2 & 0,4 & 2,6 & 0,1 & 0,5 & 0,6 & 3,0 \\
\hline
\end{tabular}

Para determinar possíveis diferenças entre os espaçamentos, após terem atendidos os pressupostos estatísticos (homogeneidade das variâncias e normalidade) os dados obtidos foram analisados por meio da análise de variância e para efeitos significativos realizou-se o teste de Scott-Knott, a 5\% de probabilidade de significância. O teste t pareado $(\alpha=5 \%)$ foi empregado para comparação entre a primeira e a segunda rotação, avaliando-se as características em cada espaçamento.

\section{Resultados e Discussão}

\section{Primeira rotação}

Verificou-se (Tabela 2) que apenas DAP e VT foram influenciados pelos espaçamentos avaliados $(\mathrm{p}<0,05)$.

Tabela 2 - Resumo da análise de variância das características diâmetro a 1,30 m de altura do solo (DAP), altura total (HT), volume total (VT), densidade básica (DB), poder calorífico superior (PCS), teor de carbono (C), teor de hidrogênio $(\mathrm{H})$ e teor de oxigênio $(\mathrm{O})$ para o clone 1277 em função do espaçamento em primeira rotação.

\begin{tabular}{lccccccccc}
\hline \multirow{2}{*}{$\begin{array}{c}\text { Fontes de } \\
\text { Variação }\end{array}$} & $\begin{array}{c}\text { Graus de } \\
\text { Liberdade }\end{array}$ & \multicolumn{7}{c}{ Quadrados Médios } \\
\cline { 3 - 10 } & & DAP & HT & VT & DB & PCS & C & H & O \\
\hline Bloco & 2 & 1,711 & 1,376 & 566,610 & 0,002 & 472,889 & 0,133 & 0,008 & 0,247 \\
Espaçamento & 5 & $4,503^{*}$ & $0,692^{\text {ns }}$ & $19743,320^{*}$ & $0,001^{\text {ns }}$ & $18012,047^{\text {ns }}$ & $0,055^{\text {ns }}$ & $0,002^{\text {ns }}$ & $0,209^{\text {ns }}$ \\
Erro & 10 & 0,246 & 0,964 & 479,882 & 0,001 & 8445,606 & 0,280 & 0,004 & 0,422 \\
\hline Coeficiente de Variação (\%) & 4,6 & 7,5 & 13,9 & 4,8 & 2,0 & 1,1 & 1,0 & 1,5 \\
\hline
\end{tabular}

Em que: * = significativo e $\mathrm{ns}=$ não significativo $(\alpha=0,05)$ pelo teste $\mathrm{F}$.

Os resultados do teste de Scott-Knott para essas características são apresentados na Tabela 3. Os maiores valores de DAP foram observados nos maiores espaçamentos ( $3,0 \mathrm{~m} \times 2,0 \mathrm{~m} ; 3,0 \mathrm{~m} \times 2,5 \mathrm{~m} \mathrm{e} 3,0 \times 3,0 \mathrm{~m})$ o que corrobora com os estudos realizados por Müller et al. (2005b), Kirongo et al. (2012) e que verificaram uma tendência de aumento de DAP com o aumento da área útil por planta.

O menor crescimento diamétrico nos menos espaçamentos por ser explicado pelo fato da competição por água, luz e nutrientes ser mais intensa nos espaçamentos mais adensados. De acordo com Lopes et al. (2017), mesmo em povoamentos jovens a concorrência entre as plantas em espaçamentos adensados é capaz de afetar o crescimento em DAP.

De acordo com Stape et al. (2010), nos espaçamentos mais adensados a proximidade dos sistemas radiculares aumentam sensivelmente a competição por água, afetando diretamente o crescimento diamétrico das plantas.

A altura total média obtida foi de $13,2 \mathrm{~m}$. Os resultados obtidos corroboram com Reiner et al. (2011) que ao avaliar o crescimento em altura de eucalipto na 
região sudoeste do Paraná, não constataram influência do espaçamento no crescimento em altura.

De modo geral, os resultados das pesquisas e plantios comerciais têm mostrado que o diâmetro é uma característica altamente responsiva aos espaçamentos, enquanto que os efeitos sobre a altura apresentam resultados controversos. Ocorrem casos onde a altura média das plantas aumenta com o aumento dos espaçamentos, e outros onde o resultado é inverso (Botelho, 1998).

Tabela 3 - Diâmetro a 1,30 m de altura do solo (DAP) e volume total (VT) para o clone 1277 em função do espaçamento em primeira rotação.

\begin{tabular}{ccc}
\hline Espaçamento & DAP (cm) & VT ( $\left.\mathbf{m}^{\mathbf{3}} \cdot \mathbf{h a}^{-\mathbf{1}}\right)$ \\
\hline $3,0 \mathrm{~m} \times 0,5 \mathrm{~m}$ & $8,8 \mathrm{c}$ & $299,2 \mathrm{a}$ \\
$3,0 \mathrm{~m} \times 1,0 \mathrm{~m}$ & $10,1 \mathrm{~b}$ & $208,6 \mathrm{~b}$ \\
$3,0 \mathrm{~m} \times 1,5 \mathrm{~m}$ & $10,6 \mathrm{~b}$ & $129,6 \mathrm{c}$ \\
$3,0 \mathrm{~m} \times 2,0 \mathrm{~m}$ & $11,1 \mathrm{a}$ & $120,8 \mathrm{c}$ \\
$3,0 \mathrm{~m} \times 2,5 \mathrm{~m}$ & $11,9 \mathrm{a}$ & $100,5 \mathrm{c}$ \\
$3,0 \mathrm{~m} \times 3,0 \mathrm{~m}$ & $12,1 \mathrm{a}$ & $87,9 \mathrm{c}$ \\
\hline
\end{tabular}

*Em que: médias seguidas da mesma letra na coluna não diferem entre ao nível de 5\% pelo teste de Scott-Knott.

Em relação ao volume total, o maior volume foi obtido no espaçamento mais adensado (3,0 $\mathrm{m}$ x 0,5 m), uma vez que espaçamentos adensados propiciam menor diâmetro nas árvores, mas apresentam maior volume por área, em função do maior número de indivíduos (maior densidade). Nos espaçamentos mais amplos (3,0 m x $2,0 \mathrm{~m} ; 3,0 \mathrm{~m} \times 2,5 \mathrm{~m} ; 3,0 \mathrm{~m} \mathrm{x} \mathrm{3,0} \mathrm{m)} \mathrm{verificou-se} \mathrm{com-}$ portamento invertido, ou seja, diâmetros com maiores dimensões e volumes individuais maiores para cada árvore, apresentando menor número de indivíduos. Guerra et al (2014) relatam que espaçamentos mais adensados atingem maiores volumes por área baseado no fato de que um maior número de plantas resulta em maior área basal e consequentemente maior volume por área.

Sereguetti et al. (2015), avaliando o efeito de nove espaçamentos em relação ao volume, observaram que em espaçamentos menores, mesmo proporcionando menor volume por planta, representaram volumes por área superiores, quando comparados aos espaçamentos mais amplos. Resultados semelhantes foram encontrados por Leite et al. (1997) que, ao avaliarem as características de crescimento de $E$. grandis aos 31 e 39 meses de idade em sete diferentes espaçamentos, verificaram que nas menores densidades de plantio ocorreram os maiores acréscimos no volume por hectare.

De acordo com Leles et al. (2001), há um contínuo decréscimo no volume por hectare de madeira em espécies de eucalipto com o aumento do espaçamento. $\mathrm{O}$ fator está relacionado ao maior volume individual fornecido por maior espaçamento de plantio, o que, consequentemente, diminui o número de indivíduos por unidade de área, resultando na diminuição da produção volumétrica total.

A densidade básica da madeira, independentemente do espaçamento utilizado, não apresentou diferenças significativas entre si, tendo o valor médio igual a 0,45 g.cm ${ }^{-3}$. Sereghetti et al. (2015), avaliando nove espaçamentos de plantio também não encontraram diferenças significativas entre o aumento do espaçamento e a densidade básica da madeira de E. urophylla x E. grandis. Embora o presente estudo e o estudo de Sereghetti et al. (2015) não tenham verificado a influência do espaçamento de plantio na densidade básica da madeira, Scolforo (1998) relata que o espaçamento influencia aspectos qualidade da madeira, como tamanho dos nós, retidão do tronco, conicidade e densidade básica.

Os espaçamentos não influenciaram o poder calorífico superior cujo valor médio foi 4598,2 Kcal.Kg1. Embora Couto et al. (2004) relatem que existe uma tendência de aumento do poder calorífico com o aumento da densidade de plantio, os resultados obtidos neste estudo corroboram com Rocha et al. (2012), que também verificaram que o espaçamento entre árvores não afetou o PCS da madeira e obtiveram resultados médios similares aos obtidos neste estudo.

Também não ocorreu efeito dos espaçamentos nos teores de $\mathrm{C}, \mathrm{H}$ e $\mathrm{O}$ na madeira, cujos valores médios foram de 48,6\%, 6,6\% e 42,1\%, respectivamente. Os valores obtidos estão próximos aos obtidos por Klock et al. (2005) que relatam valores de $49-50 \%$ para C, $6 \%$ para $\mathrm{H}$ e $44-45 \%$ para $\mathrm{O}$.

Geralmente, o rendimento médio em carbono para clones de eucalipto, segundo Trugilho et al. (2005), é em torno de $26 \%$, valores inferiores aos encontrados nesse estudo. Segundo Paula et al. (2011) e Protásio et al. (2011), um acréscimo no teor de oxigênio e uma redução do teor de hidrogênio tendem a diminuir o poder calorífico de combustíveis de biomassa vegetal. 


\section{Segunda rotação}

Na segunda rotação, foi observado efeito signi-

ficativo dos espaçamentos para DAP, VT e teores de O, C e H (Tabelas 4 e 5).

Tabela 4 - Resumo da análise de variância das características diâmetro a 1,30 m de altura do solo (DAP), altura total (HT), volume total (VT), densidade básica (DB), poder calorífico superior (PCS), teor de carbono (C), teor de hidrogênio $(\mathrm{H})$ e teor de oxigênio $(\mathrm{O})$ para o clone 1277 em função do espaçamento em segunda rotação.

\begin{tabular}{lccccccccc}
\hline \multirow{2}{*}{$\begin{array}{c}\text { Fontes de } \\
\text { Variação }\end{array}$} & \multirow{2}{*}{$\begin{array}{c}\text { Graus de } \\
\text { Liberdade }\end{array}$} & \multicolumn{7}{c}{ Quadrados Médios } \\
\cline { 2 - 10 } & & DAP & HT & VT & DB & PCS & C & H & O \\
\hline Bloco & 2 & 11,274 & 14,541 & 2078,616 & $3,9.10^{-5}$ & 562,065 & 8,011 & 0,239 & 10,364 \\
Espaçamento & 5 & $7,048^{*}$ & $7,021^{\text {ns }}$ & $5562,809^{*}$ & $2,2.10^{-5}$ ns & $1251,689^{\text {ns }}$ & $13,633^{*}$ & $0,615^{*}$ & $19,326^{*}$ \\
Erro & 10 & 1,259 & 3,872 & 266,472 & $4,6.10^{-5}$ & 9667,690 & 2,677 & 0,098 & 3,357 \\
Coeficiente de Variação (\%) & 15,2 & 17,2 & 15,9 & 1,4 & 2,1 & 3,6 & 5,6 & 3,8 \\
\hline
\end{tabular}

Em que: * = significativo e $\mathrm{ns}=$ não significativo $(\alpha=0,05)$ pelo teste $\mathrm{F}$.

Tabela 5 - Diâmetro a 1,30 m de altura do solo (DAP) e teor de oxigênio (O), carbono (C) e hidrogênio (H) na madeira para o clone 1277 em função do espaçamento em segunda rotação.

\begin{tabular}{cccccc}
\hline Espaçamento & DAP (cm) & VT $\left(\mathbf{m}^{3} \cdot \mathbf{h a}^{-1}\right)$ & C (\%) & H (\%) & O (\%) \\
\hline $3,0 \mathrm{~m} \times 0,5 \mathrm{~m}$ & $5,0 \mathrm{~b}$ & $172,7 \mathrm{a}$ & $44,3 \mathrm{~b}$ & $5,5 \mathrm{~b}$ & $49,3 \mathrm{a}$ \\
$3,0 \mathrm{~m} \times 1,0 \mathrm{~m}$ & $6,5 \mathrm{~b}$ & $135,2 \mathrm{~b}$ & $46,8 \mathrm{a}$ & $5,9 \mathrm{a}$ & $46,5 \mathrm{~b}$ \\
$3,0 \mathrm{~m} \times 1,5 \mathrm{~m}$ & $7,0 \mathrm{~b}$ & $81,8 \mathrm{c}$ & $46,5 \mathrm{a}$ & $6,0 \mathrm{a}$ & $46,8 \mathrm{~b}$ \\
$3,0 \mathrm{~m} \times 2,0 \mathrm{~m}$ & $8,0 \mathrm{a}$ & $98,6 \mathrm{c}$ & $41,7 \mathrm{~b}$ & $5,0 \mathrm{~b}$ & $52,5 \mathrm{a}$ \\
$3,0 \mathrm{~m} \times 2,5 \mathrm{~m}$ & $8,7 \mathrm{a}$ & $69,9 \mathrm{c}$ & $47,0 \mathrm{a}$ & $6,1 \mathrm{a}$ & $46,2 \mathrm{~b}$ \\
$3,0 \mathrm{~m} \times 3,0 \mathrm{~m}$ & $9,1 \mathrm{a}$ & $58,7 \mathrm{c}$ & $43,7 \mathrm{~b}$ & $5,3 \mathrm{~b}$ & $50,3 \mathrm{a}$ \\
\hline
\end{tabular}

* Em que: médias seguidas da mesma letra na coluna não diferem entre ao nível de 5\% pelo teste de Scott-Knott.

Assim como na primeira rotação, o DAP foi influenciado pelos espaçamentos, apresentando maiores valores nos espaçamentos menos adensados $(3,0 \mathrm{~m} \mathrm{x}$ $2,0 \mathrm{~m} ; 3,0 \mathrm{~m} \times 2,5 \mathrm{~m}$ e $3,0 \times 3,0 \mathrm{~m}$ ), corroborando com Müller et al. (2005b), que observaram uma tendência de aumento do DAP com o aumento da área útil por planta.

A altura total das árvores também não foi influenciada pelos espaçamentos na segunda rotação, sendo a altura média igual a $11,4 \mathrm{~m}$. Para volume total, o resultado foi similar ao obtido para a primeira rotação, ou seja, o maior valor foi encontrado no espaçamento

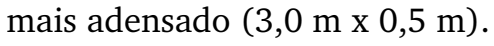

Os espaçamentos também não influenciaram a densidade média da madeira na segunda rotação cujo valor médio foi de $0,47 \mathrm{~g} . \mathrm{cm}^{-3}$. Valores similares foram encontrados por Valle (2009) ao avaliar plantações comerciais de E. urophylla, obtendo densidade média de 0,48 g. $\mathrm{cm}^{-3}$ na segunda rotação. De acordo com Carneiro et al. (2014), altos valores de densidade básica são desejáveis para a utilização energética da madeira uma vez que esta característica relaciona-se diretamente com a produção de energia, sendo um dos principais critérios para seleção de espécies/clones para a queima direta. Entretanto, Lopes (2017) ressalta que deve-se observar que esse não é o único parâmetro de avaliação do potencial de um determinado clone para uso energético.

O poder calorífico superior cujo valor médio foi $4478,5 \mathrm{Kcal} \mathrm{Kg}^{-1}$ não foi influenciado pelos espaçamentos. Melo (2016), avaliando o poder calorífico superior de $E$. grandis em segunda rotação, obteve valor médio de 17,62 MJ, o que equivale a 4208,5 Kcal. $\mathrm{Kg}^{-1}$.

Os teores de carbono, hidrogênio e oxigênio na madeira foram influenciados pelos espaçamentos na segunda rotação, não sendo possível observar uma tendência entre os espaçamentos e os teores desses componentes elementares da madeira.

Os teores de $\mathrm{C}$ e $\mathrm{H}$ foram maiores nos espaçamentos $3,0 \mathrm{~m} \mathrm{x} 1,0 \mathrm{~m} ; 3,0 \mathrm{~m} \times 1,5 \mathrm{~m}$ e $3,0 \mathrm{~m} \mathrm{x} \mathrm{2,5} \mathrm{m.}$ Santos et al. (2012) ressaltam que maiores teores de carbono são desejáveis quando se objetiva a produção de carvão vegetal ou a queima direta da madeira uma 
Guimarães, V. M. et al.

vez os maiores teores de carbono propiciam uma maior capacidade térmica em função da maior energia liberada.

Segundo Behling et al. (2014), a influência causada pelo espaçamento em relação ao teor de carbono entre as árvores está associada ao diferente tamanho que as árvores apresentam, característica que sofre influência pelo espaçamento entre plantas e, também, associada às condições de crescimento que são impostas por esse fator. De acordo com Stape et al. (2001), árvores cultivadas em espaçamentos adensados podem maximizar a intercepção da radiação solar, o armazenamento de carbono e aumentar a produção de biomassa para energia.

Em relação ao hidrogênio, Protásio et al. (2011) relatam que, na composição da madeira, baixos valores de hidrogênio resultam em uma alta relação $\mathrm{C} / \mathrm{H}$, fator que é indesejável quando se busca a produção de energia, visto que pequenos acréscimos no teor de hidrogênio promovem um elevado ganho no valor calórico dos combustíveis de biomassa.

Observou que os espaçamentos $3,0 \mathrm{~m} \times 0,5 \mathrm{~m}$; $3,0 \mathrm{~m} \times 2,0 \mathrm{~m}$ e 3,0 $\mathrm{m} \times 3,0 \mathrm{~m}$ foram os que apresentaram maiores teores de O na madeira. Ragland et al. (1991) relatam que o teor de $\mathrm{O}$ na madeira é da ordem de 40 a $44 \%$, sendo que os valores obtidos neste estudo foram superiores ao relatado.

\section{Comparação entre as duas rotações}

Os resultados da comparação das características analisadas entre os espaçamentos nas duas rotações são apresentados nas Tabelas 6, 7 e 8 .

Tabela 6 - Valores médios para as variáveis dendrométricas para o clone 1277, em função do espaçamento, em primeira (R1) e segunda (R2) rotação, e resultado do teste t para comparação entre as duas rotações*.

\begin{tabular}{cccccccccc}
\hline \multirow{2}{*}{ Espaçamento } & \multicolumn{3}{c}{ DAP } & \multicolumn{3}{c}{ HT } & \multicolumn{3}{c}{ VT } \\
\cline { 2 - 10 } & R1 & R2 & t & R1 & R2 & t & R1 & R2 & t \\
\hline $3,0 \mathrm{~m} \times 0,5 \mathrm{~m}$ & 8,8 & 5,0 & $3,16^{*}$ & 12,7 & 9,8 & $1,83^{\text {ns }}$ & 299,2 & 172,7 & $3,03^{*}$ \\
$3,0 \mathrm{~m} \times 1,0 \mathrm{~m}$ & 10,1 & 6,5 & $2,79^{*}$ & 13,2 & 10,3 & $1,42^{*}$ & 208,6 & 135,2 & $3,57^{*}$ \\
$3,0 \mathrm{~m} \times 1,5 \mathrm{~m}$ & 10,6 & 7,0 & $2,85^{*}$ & 12,9 & 11,1 & $0,86^{*}$ & 129,6 & 98,6 & $4,79^{*}$ \\
$3,0 \mathrm{~m} \times 2,0 \mathrm{~m}$ & 11,1 & 9,1 & $1,72^{*}$ & 12,7 & 14,2 & $1,22^{*}$ & 120,8 & 81,8 & $1,60^{*}$ \\
$3,0 \mathrm{~m} \times 2,5 \mathrm{~m}$ & 11,9 & 8,7 & $2,15^{*}$ & 13,3 & 11,6 & $1,28^{*}$ & 100,5 & 69,9 & $2,64^{*}$ \\
$3,0 \mathrm{~m} \times 3,0 \mathrm{~m}$ & 12,1 & 8,0 & $2,20^{*}$ & 14,0 & 11,6 & $1,37^{*}$ & 87,9 & 58,7 & $2,27^{*}$ \\
\hline
\end{tabular}

* Em que: DAP = diâmetro 1,30 $\mathrm{m}$ do solo $(\mathrm{cm}), \mathrm{HT}=$ altura total $(\mathrm{m}), \mathrm{VT}=$ volume total $\left(\mathrm{m} 3 \cdot \mathrm{ha}^{-1}\right)$, * $=$ significativo, ns $=$ não significativo pelo teste $\mathrm{t}(\alpha=0,05)$.

O DAP e o VT diferiram, em todos os espaçamentos, nas duas rotações, sendo que a HT não foi similar nas duas rotações apenas no espaçamento $3,0 \mathrm{~m}$ x $0,5 \mathrm{~m}$ (Tabela 6). Verificou-se que houve redução nas variáveis dendrométricas da primeira para a segunda rotação. Em termos médios, os valores foram de $31,5 \%, 13,6 \%$, e $34,8 \%$, respectivamente, para DAP, HT e VT.

A queda de produtividade da primeira para a segunda rotação tem sido frequentemente observada em povoamentos de eucalipto (Faria et al., 2002). Fatores relacionados a qualidade do material genético, altura e época de corte das cepas, características edafoclimáticas do local e tratos silviculturais, entre outros influenciam na capacidade de rebroto e desenvolvimento de novos fustes afetando a produtividade (Silva e Angeli, 2006).

Ressalta-se que, em algumas situações, de acordo com Gonçalves et al. (2014), obtêm-se produção semelhante ou até mesmo superiores na segunda rotação. No povoamento em estudo, provavelmente, a redução da produtividade pode estar atrelada ao fato do adensamento que provou uma maior competitividade entre as árvores.
Analisando a densidade básica da madeira, apenas nos espaçamentos 3,0 m x 1,5 m e 3,0 m x 2,5 m não ocorreram diferenças da primeira para a segunda rotação, sendo observado, para os demais espaçamentos, um acréscimo no valor da densidade observada na segunda rotação. Valle (2009) também observou acréscimo na DB na segunda rotação em comparação à primeira.

O PCS foi similar nas duas rotações apenas no espaçamento mais adensado, ou seja, 3,0 m x 0,5 m, sendo que, em termos médios, ocorreu um aumento de $1,8 \%$ na segunda rotação.

Comportamento similar ao obtido para PCS foi verificado para os teores de $\mathrm{C}, \mathrm{H}$ e $\mathrm{O}$, ou seja, apenas no espaçamento mais adensado os valores foram similares nas duas rotações. Verificou-se que houve queda nos teores de carbono e hidrogênio. Para o oxigênio, houve um acréscimo no seu teor, sendo os valores médios de $7,4 \%, 15,1 \%$ e $15,4 \%$ respectivamente. 
Guimarães, V. M. et al.

Tabela 7 - Valores médios para as características da madeira para o clone 1277, em função do espaçamento, em primeira (R1) e segunda (R2) rotação, e resultado do teste t para comparação entre as duas rotações*.

\begin{tabular}{ccccccc}
\hline \multirow{2}{*}{ Espaçamento } & \multicolumn{7}{c}{ DB } & \multicolumn{5}{c}{ PCS } \\
\cline { 2 - 7 } & R1 & R2 & t & R1 & R2 & t \\
\hline $3,0 \mathrm{~m} \times 0,5 \mathrm{~m}$ & 0,44 & 0,47 & $3,49^{*}$ & 4713,7 & 4687,5 & $0,47^{\text {ns }}$ \\
$3,0 \mathrm{~m} \times 1,0 \mathrm{~m}$ & 0,44 & 0,47 & $1,04^{*}$ & 4552,3 & 4657,1 & $1,84^{*}$ \\
$3,0 \mathrm{~m} \times 1,5 \mathrm{~m}$ & 0,47 & 0,47 & $0,12^{\mathrm{ns}}$ & 4679,2 & 4714,2 & $2,22^{*}$ \\
$3,0 \mathrm{~m} \times 2,0 \mathrm{~m}$ & 0,44 & 0,47 & $1,40^{*}$ & 4541,3 & 4663,8 & $0,91^{*}$ \\
$3,0 \mathrm{~m} \times 2,5 \mathrm{~m}$ & 0,47 & 0,47 & $0,10^{\mathrm{ns}}$ & 4565,5 & 4677,3 & $1,27^{*}$ \\
$3,0 \mathrm{~m} \times 3,0 \mathrm{~m}$ & 0,45 & 0,48 & $1,18^{*}$ & 4537,2 & 4670,9 & $7,05^{*}$ \\
\hline
\end{tabular}

* Em que: DA = densidade básica $\left(\mathrm{g} . \mathrm{cm}^{-3}\right), \mathrm{PCS}=$ poder calorífico superior $\left(\mathrm{Kcal}_{\mathrm{Kg}}{ }^{-1}\right)$, * = significativo, ns $=$ não significativo pelo teste $\mathrm{t}(\alpha=0,05)$.

Tabela 8 - Valores médios para os componentes elementares da madeira para o clone 1277, em função do espaçamento, em primeira (R1) e segunda (R2) rotação, e resultado do teste t para comparação entre as duas rotações*.

\begin{tabular}{|c|c|c|c|c|c|c|c|c|c|}
\hline \multirow{2}{*}{ Espaçamento } & \multicolumn{3}{|c|}{ C } & \multicolumn{3}{|c|}{$\mathbf{H}$} & \multicolumn{3}{|c|}{$\mathbf{O}$} \\
\hline & R1 & R2 & $\mathbf{t}$ & R1 & R2 & $\mathbf{t}$ & R1 & R2 & $\mathbf{t}$ \\
\hline $3,0 \mathrm{~m} \times 0,5 \mathrm{~m}$ & 48,6 & 44,4 & $1,66^{\mathrm{ns}}$ & 6,7 & 5,5 & $2,0^{\mathrm{ns}}$ & 41,8 & 49,3 & $2,12^{\mathrm{ns}}$ \\
\hline $3,0 \mathrm{~m} \times 1,0 \mathrm{~m}$ & 48,4 & 46,8 & $0,85^{*}$ & 6,6 & 6,0 & $1,63^{*}$ & 42,4 & 46,5 & $1,77^{*}$ \\
\hline $3,0 \mathrm{~m} \times 1,5 \mathrm{~m}$ & 48,6 & 46,5 & $1,79^{*}$ & 6,6 & 6,0 & $2,64^{*}$ & 42,3 & 46,8 & $2,92^{*}$ \\
\hline $3,0 \mathrm{~m} \times 2,0 \mathrm{~m}$ & 48,8 & 41,7 & $3,55^{*}$ & 6,6 & 5,0 & $4,57^{*}$ & 41,8 & 52,6 & $3,73^{*}$ \\
\hline $3,0 \mathrm{~m} \times 2,5 \mathrm{~m}$ & 48,5 & 47,0 & $1,33^{*}$ & 6,6 & 6,1 & $2,39^{*}$ & 42,1 & 46,2 & $2,43^{*}$ \\
\hline $3,0 \mathrm{~m} \times 3,0 \mathrm{~m}$ & 48,6 & 43,7 & $4,31^{*}$ & 6,6 & 5,3 & $5,49^{*}$ & 42,3 & 50,3 & $5,40^{*}$ \\
\hline
\end{tabular}

* Em que: $\mathrm{C}$ = teor de carbono (\%), $\mathrm{H}=$ teor de hidrogênio (\%), $\mathrm{O}=$ teor de oxigênio (\%), * = significativo, ns = não significativo pelo teste $\mathrm{t}$ $(\alpha=0,05)$.

Segundo Demirbas (2004), a avaliação do percentual de carbono é um parâmetro importante para analisar o potencial energético da biomassa vegetal, sendo que pequenas mudanças nessa característica, podem contribuir para um acréscimo considerável do poder calorífico. Verificou-se, portanto, com exceção para o espaçamento mais adensado, que em relação aos teores dos componentes elementares ( $\mathrm{C} \mathrm{e} \mathrm{H}$ ) da madeira, a segunda rotação apresentou menor potencial para uso energético quando comparada com a primeira rotação.

De acordo com Reis et al. (2012), identifica-se uma tendência entre hidrogênio e carbono, a diminuição do rendimento em carbono está associada com o aumento no teor de hidrogênio presente na biomassa vegetal. Quando o objetivo é a produção energética, devem ser escolhidas madeiras com baixos teores de hidrogênio. Já o teor de oxigênio, segundo Nordin (1994) e Demirbas (1997), expõe relação negativa com o poder calorífico, o aumento no teor de oxigênio da biomassa possui a tendência de reduzir o valor calórico.
Ressalta-se que, na maioria dos casos, o sistema talhadia é viável tecnicamente, porém, faz-se necessária a avaliação econômica para a decisão final da condução do povoamento em talhadia ou reforma do mesmo.

Considerando como objetivo a produção energética e as condições edafoclimáticas de Jataí-GO assim como a produção volumétrica e o poder calorífico superior, o manejo em duas rotações com uso do espaçamento 3,0 $\mathrm{m}$ x $0,5 \mathrm{~m}$ em curta rotação apresenta-se como o mais indicado.

\section{Conclusões}

Na primeira rotação, apenas o DAP e o VT foram influenciados pelos espaçamentos enquanto que na segunda rotação, os espaçamentos influenciaram o DAP, o VT e os teores de C, $\mathrm{H}$ e O.

As características dendrométricas apresentaram decréscimo na segunda rotação enquanto DB e PCS apresentaram acréscimos. Os teores de $\mathrm{C}, \mathrm{H}$ e $\mathrm{O}$ não apresentaram tendência entre os espaçamentos. 


\section{Referências}

Alvarez, C. A.; Stape, J. L.; Sentelhas, P. C.; Gonçalves, J. L. M.; Sparovek, G. 2013. Köppen's climate classification map for Brazil. Metorologische Zeitschrift, 22: 711-728. Doi: https://doi.org/10.1127/09412948/2013/0507.

Associação Brasileira de Normas Técnicas - ABNT. 1984. NBR 8633: carvão vegetal - determinação do poder calorífico. ABNT, Rio de Janeiro.

Associação Brasileira de Normas Técnicas - ABNT. 2003. NBR 11941: madeira - determinação da densidade básica. ABNT, Rio de Janeiro.

Barros, N. F.; Faria, G. E.; Novaes, R. F.; Lima, J. C.; Teixeira, J. L. 2002. Produção e estado nutricional de povoamentos de Eucalyptus grandis, em segunda rotação, em resposta à adubação potássica. Revista Árvore, 26: $577-584$

Bech, N.; Jensen, P. A.; Dam-Johansen, K. 2009. Determining the elemental composition of fuels by bomb calorimetry and the inverse correlation of HHV with elemental composition. Biomass and Bioenergy, 33: 534-537. Doi: https://doi.org/10.1016/j.biombioe.2008.08.015.

Behling, A.; Sanquetta, C. R.; Caron, B. O.; Schmidt, D.; Elli, E. F.; Corte, A. P. D. 2014. Teores de carbono orgânico de três espécies arbóreas em diferentes espaçamentos. Pesquisa Florestal Brasileira, 34: 13-19. Doi: https://doi.org/10.4336/2014.pfb.34.77.562.

Botelho, S. A. 1998. Espaçamento. p. 381-405. In: Scolforo, J. R. S. Manejo florestal. UFLA/FAEPE, Lavras.

Campos, J. C. C.; Leite, H. G. 2017. Mensuração florestal: perguntas e respostas. 5.ed. UFV, Viçosa.

Carneiro, A. C. O.; Castro, A. F. N. M.; Castro, R. V. O.; Santos, R. C.; Ferreira, L. P.; Damásio, R. A. P.; Vital, B. R. 2014. Potencial energético da madeira de Eucalyptus sp. em função da idade e de diferentes materiais genéticos. Revista Árvore, 38: 375-381. Doi: http://dx.doi. org/10.1590/S0100-67622014000200019.

Couto, L.; Müller, M. D.; Tsukamoto, A. A.; Barcellos, D. C.; Fonseca, Ê. M. B.; Corrêa, M. R. 2004. Programa de pesquisa para avaliação de densidades de plantio e rotação de plantações de rápido crescimento para produção de biomassa. Biomassa \& Energia, 1:107-112.

Couto, L.; Müller, M. D. 2008. Florestas energéticas no Brasil. p. 93 111. In: Cortez, L. A. B.; Lora, E. E. S.; Gómez, E. O. Biomassa para energia. Unicamp, Campinas.

Crous, J. W.; Burger, L. 2014. A comparison of planting and coppice regeneration of Eucalyptus grandis $\times$ Eucalyptus urophylla clones in South Africa. Southern Forests, 77: 277-285. Doi: https://doi.org/10 $.2989 / 20702620.2015 .1063031$

Demirbas, A. 1997. Calculation of higher heating values of biomass fuels. Fuel, 76: 431-434. Doi: https://doi.org/10.1016/S00162361(97)85520-2.

Demirbas, A. 2004. Combustion characteristics of different biomass fuels. Progress in Energy and Combustion Science, 30: 219-230. Doi: https://doi.org/10.1016/j.pecs.2003.10.004.

Empresa Brasileira de Pesquisa Agropecuária - Embrapa. 2018. Sistema brasileiro de classificação de solos. 5 ed. EMBRAPA-SPI, Brasília.

Evans, J.; Turnbull, J. W. 2004. Plantation forestry in the tropics: the role, silviculture, and use of planted forest for industrial, social, environmental, and agroforestry. 3.rd. Oxford University Press, Oxford.
Faria, G. E.; Barros, N. F; Novais, R. F.; Lima, J. C.; Teixeira, J. L. 2002. Produção e estado nutricional de povoamentos de Eucalyptus grandis, em segunda rotação, em resposta à adubação potássica. Revista Árvore, 26: $577-584$.

Gonçalves, J. L. M.; Alvares, C. A.; Behling, M.; Alves, J. M.; Pizzi, G. T.; Angeli, A. 2014. Produtividade de plantações de eucalipto manejadas nos sistemas de alto fuste e talhadia, em função de fatores edafoclimáticos. Scientia Florestalis, 42: 411-419.

Guedes, I. C. L.; Coelho Júnior, L. M.; Oliveira, A. D.; Mello, J. M.; Rezende, J. L. P.; Silva, C. P. C. 2011. Economic analysis of replacement regeneration and coppice regeneration in eucalyptus stands under risk conditions. Cerne, 17: 393-401. Doi: http://dx.doi.org/10.1590/ S0104-77602011000300014.

Guerra, S. P. S.; Garcia, E. A.; Lanças, K. P.; Rezende, M. A.; Spinelli, R. 2014. Heating value of eucalypt wood grown on SRC for energy production. Fuel, 137: 360-363. Doi: https://doi.org/10.1016/j. fuel.2014.07.103.

Indústria Brasileira de Árvores - IBÁ. 2017. Relatório 2017. Disponível em: https://www.iba.org/publicacoes/relatorios.

Indústria Brasileira de Árvores - IBÁ. 2019. Relatório 2019. Disponível em: https://www.iba.org/publicacoes/relatorios.

Kirongo, B. B.; Mbelase, A. S.; Senelwa, K.; Hitimana, J.; Etiegni, L. 2012. Spacing and genotype on height and diameter growth of four eucalyptus under short rotation. Jurnal Manajemen Hutan Tropika, 18: 1-9. Doi: https://doi.org/10.7226/jtfm.18.1.1.

Klock, V.; Muñiz, G. I. B.; Hernandez, J. A.; Andrade, A. S. 2005. Química da madeira. 3.ed. UFPR, Curitiba. Disponível em: https:// bit.ly/2SqOSOp.

Leite, F. P.; Barros, N. F.; Novais, R. F.; Sans, L. M. A.; Fabres, A. S. 1997. Crescimento de Eucalyptus grandis em diferentes densidades populacionais. Revista Árvore, 21: 313-321.

Leles, P. S. S.; Reis, G. G.; Reis, M. G. F.; Morais, E. J. 2001. Crescimento, produção e alocação de matéria seca de Eucalyptus camaldulensis e Eucalyptus pellita sob diferentes espaçamentos na região de cerrado, MG. Scientia Forestalis, 59: 77-87.

Lopes, E. D. 2017. Avaliação de clones de Eucalyptus spp e Corymbia spp em diferentes espaçamentos visando à produção de bioenergia. Diamantina: Universidade Federal dos Vales do Jequitinhonha e Mucuri, 120f. Tese Doutorado. Disponível em: https://bit.ly/2CykXPL.

Lopes, E. D.; Laia, M. L.; Santos, A. S.; Soares, G. M.; Leite, R. W. P.; Martins, N. S. 2017. Influência do espaçamento de plantio na produção energética de clones de Corymbia e Eucalyptus. Floresta, 47: 95-104. Doi: http://dx.doi.org/10.5380/rf.v47i1.47141.

Macedo, I. C. 2003. Estado da arte e tendências tecnológicas para energia CTEnerg, Brasília. Disponível em: https://bit.ly/2S9ayiM.

Melo, R. X. 2016. Avaliação de um sistema florestal de curta rotação de Eucalyptus spp. em função da desbrota e adubação. Botucatu: Universidade Estadual Paulista, 52f. Dissertação Mestrado. Disponível em: http://hdl.handle.net/11449/138336.

Müller, M. D.; Couto, L.; Neves, J. C. L. 2005a. Produção de biomassa e balanço nutricional de plantações de eucalipto clonal em diferentes densidades de plantio no município de Itamarandiba-MG. Biomassa \& Energia, 2: 91-101. 
Guimarães, V. M. et al.

Müller, M. D.; Couto, L.; Leite, H. G.; Brito, J. O. 2005b. Avaliação de um clone de eucalipto estabelecido em diferentes densidades de plantio para produção de biomassa e energia. Biomassa \& Energia, 2: 177-186.

Nordin, A. 1994. Chemical elemental characteristics of biomass fuels. Biomass and Bioenergy, 6: 339-347. Doi: https://doi.org/10.1016/09619534(94)E0031-M.

Paixão, F. A.; Soares, C. P. B.; Jacovine, L. A. G.; Silva, M. L.; Leite, H. G.; Silva, G. F. 2006. Quantificação do estoque de carbono e avaliação econômica de diferentes alternativas de manejo em um plantio de eucalipto. Revista Árvore, 30: 411-420.

Paula, L. E. R.; Trugilho, P. F.; Napoli, A.; Bianchi, M. L. 2011. Characterization of residues from plant biomass for use in energy generation. Cerne, 17: 237-246. Doi: http://dx.doi.org/10.1590/ S0104-77602011000200012.

Prevedello, J.; Kaiser, D. R.; Reinert, D. J.; Vogelmann, E. S.; Fontanela, E.; Reichert, J. M. 2013. Manejo do solo e crescimento inicial de Eucalyptus grandis Hill ex Maiden em argissolo. Ciência Florestal, 23: 129-138. Doi: http://dx.doi.org/10.5902/198050988447.

Protásio, T. P.; Bufalino, L.; Tonoli, G. H. D.; Couto, A. M.; Trugilho, P. F; Guimarães Júnior, M. 2011. Relação entre o poder calorífico superior e os componentes elementares e minerais da biomassa vegetal. Pesquisa Florestal Brasileira, 31: 122-133. Doi: http://dx.doi.org/10.4336/2011. pfb.31.66.113.

Protásio, T. P.; Neves, T. A.; Reis, A. A.; Trugilho, P. F. 2014. Efeito da idade e clone na qualidade da madeira de Eucalyptus spp. visando à produção de bioenergia. Ciência Florestal, 24: 465-477. Doi: http:// dx.doi.org/10.5902/1980509814587.

Ragland, K. W.; Aerts, D. J.; Baker, A. J. 1991. Properties of wood for combustion analysis. Bioresource Technology, 37: 161-168. Doi: https:// doi.org/10.1016/0960-8524(91)90205-X.

Reiner, D. A.; Silveira, E. R.; Szabo, M. S. 2011. O uso do eucalipto em diferentes espaçamentos como alternativa de renda e suprimento da pequena propriedade na região do sudeste do Paraná. Synergismus Scyentífica, 6: 1-7.

Reis, A. A.; Protásio, T. P.; Melo, I. C. N. A.; Trugilho, P. F; Carneiro, A. C. O. 2012. Composição da madeira e do carvão vegetal de Eucalyptus urophylla em diferentes locais de plantio. Pesquisa Florestal Brasileira, 32: 277-290. Doi: https://doi.org/10.4336/2012.pfb.32.71.277.

Rocha, M. F. V.; Vital, B. R.; Carneiro, A. C. O.; Carvalho, A. M. M. L.; Nogueira, G. S.; Couto, L. 2012. Efeito da idade e do espaçamento nas propriedades da madeira para energia. Biomassa \& Energia, 5: 27-33.
Santos, L. C.; Carvalho, A. M. M. L.; Pereira, B. L. C.; Oliveira, A. C.; Carneiro, A. C. O.; Trugilho, P. F. 2012. Propriedades da madeira e estimativas de massa, carbono e energia de clones de Eucalyptus plantados em diferentes locais. Revista Árvore, 36: 971-980. Doi: https://doi.org/10.1590/S0100-67622012000500019.

Scolforo, J. R. S. 1998. Manejo florestal. UFLA/FAEPE, Lavras.

Scolforo, J. R. S. 2008. O mundo eucalipto: os fatos e mitos de sua cultura. Mar de Ideias, Rio de Janeiro.

Sereghetti, G. C.; Lanças, K. P.; Sartori, M. S.; Rezende, M. A.; Soler, R. R. 2015. Efeito do espaçamento no crescimento e na densidade básica da madeira de Eucalyptus urophylla x Eucalyptus grandis em florestas de ciclo curto. Energia na Agricultura, 30: 257-262. Doi: https://doi. org/10.17224/EnergAgric.2015v30n3p257-262.

Silva, P. H. M.; Angeli, A. 2006. Implantação e manejo de florestas comerciais. Disponível em: https://bit.ly/3791LBm.

Stape, J. L.; Binkley, D.; Ryan, M. G.; Fonseca, R. A.; Loos; R. A.; Takahashi, E. N.; Silva, C. R.; Silva, S. R.; Hakamada, R. E.; Ferreira, J. M. A.; Lima, A. M. N.; Gava, J. L.; Leite, F. P; Andrade, H. B.; Alves, J. M.; Silva, G. G. C.; Azevedo, M. R. 2010. The Brazil eucalyptus potential productivity project: influence of water, nutrients and stand uniformity on wood production. Forest Ecology and Management, 259: 1684-1694. Doi: https://doi.org/10.1016/j.foreco.2010.01.012.

Stape, J. L.; Gonçalves, J. L. M.; Gonçalves, A. N. 2001. Relationships between nursery practices and field performance for Eucalyptus plantations in Brazil. New Forests, 22: 19-41. Doi: https://doi. org/10.1023/A:1012271616115.

Trugilho, P. F; Silva, J. R. M.; Mori, F. A.; Lima, J. T.; Mendes, L. M.; Mendes, L. F. B. 2005. Rendimentos e características do carvão vegetal em função da posição radial de amostragem em clones de Eucalyptus. Cerne, 11: 178-186.

Valle, M. L. A. 2009. Propriedades da madeira de eucalipto de primeira e segunda rotação, visando a sua utilização como madeira preservada. Viçosa: Universidade Federal de Viçosa. 80f. Dissertação Mestrado em Ciência Floresta. Disponível em: https://bit.ly/39h6AtV.

Vechi, A.; Magalhães Júnior, C. A. O. 2018. Aspectos positivos e negativos da cultura do eucalipto e os efeitos ambientais do seu cultivo. Revista Valore, 3: 495-507. Doi: https://doi.org/10.22408/reva312018101495507. 